\title{
La Revue Officielle de la SFED \\ ou « Acta Endoscopica, un périodique de plus ou quelque chose d'autre »?
}

\section{Heresbach}

Après le changement de direction de la revue Acta Endoscopica fin 2008, la destinée de la revue s'est posée tant sur la forme que sur le fond. À l'heure de l'hyperspécialisation parfois érigée en règle de bonne pratique, de la multitude des sources de formations, et confrontée à la diversité des informations parfois contradictoires, la Société Française d'Endoscopie Digestive (SFED) a décidé parmi les différentes possibilités, de prendre en charge la continuité de parution de cette revue.

Dès lors, le choix de la ligne éditoriale reposait sur la nécessité de balayer les différents aspects de la spécialité (endoscopie diagnostique, endoscopie thérapeutique, endoscopie sans tube par capsule) ainsi que les questions générées par les différents modes d'exercice bien au-delà du classique clivage privé-public, s'intéressant plutôt aux modalités pratiques d'exercice, basées sur la définition du périmètre de la spécialité et de son support technologique qu'est un plateau technique d'endoscopie. Ces choix ont été effectués, conscients que la renommée d'une revue n'est souvent jugée que par le niveau d'impact factor, en particulier par un cercle d'initiés, alors que sa diffusion, et donc son utilité, est davantage basée sur la pertinence des informations médicales et professionnelles qu'elle publie. Sa crédibilité repose sur la rigueur intégrant la qualité et la ponctualité de l'information diffusée mais également sur la pondération des recommandations scientifiques à l'heure de l'Evidence Based Medicine par les contraintes et réalités de l'exercice quotidien faisant intervenir de nombreux facteurs, en particulier humains, en endoscopie digestive.

D. Heresbach $(\bowtie)$

Rédacteur en chef

Commission recommandation de la SFED

Service des Maladies de l'Appareil Digestif, CHU Pontchaillou,

F-35033 Rennes Cedex 9, France

E-mail : denis.heresbach@chu-rennes.fr
Nous proposons donc que la Revue Officielle de la Société Française d'Endoscopie Digestive creuse son sillon progressivement, associant la publication d'articles originaux ou de cas cliniques tout en laissant une large part à l'information professionnelle sous la forme de dossiers thématiques, d'informations techniques basées sur l'interview d'experts, d'images commentées classiques ou rares, d'une publication régulière des recommandations de la SFED ou de celles émises par d'autres sociétés savantes, d'une veille scientifique sous l'aspect d'une revue de presse et de congrès mais également d'une veille professionnelle et institutionnelle plus générale en y incluant les informations professionnelles habituellement publiées dans la lettre de la SFED.

La qualité d'une revue professionnelle repose également sur sa présence à des rendez-vous fixes qui jalonnent notre exercice, et la possibilité de transmettre les informations de façon concomitante aux congrès et réunions scientifiques qui ponctuent la vie de la Société Française d'Endoscopie Digestive et des gastroentérologues pratiquant l'endoscopie digestive. Ainsi, chaque numéro paraîtra lors de ces évènements en incluant les textes des communications ou un résumé commenté des présentations, en particulier du printemps lors du Symposium SFED des JFHOD, des Universités d'été d'endoscopie digestive, et à l'automne du cours de Vidéodigest, des journées du CREGG et de la réunion de la SMIER.

Le comité éditorial reste naturellement ouvert à toute suggestion permettant d'améliorer la diffusion de l'information professionnelle, en souhaitant que la Revue Officielle de la SFED vous permette de retrouver, de façon unifiée sur un même support, la majorité des informations concernant l'exercice de l'endoscopie digestive. Loin de la situation de crise actuelle et sans verser dans une « endoscopica-mania », le comité éditorial sous l'égide de la SFED a décidé d'adopter le «Yes we can ».

Bonne lecture. 